\section{RADIO REFLEXIONS FROM METEORIC IONIZATION} T a meeting of the Physical Society held on
January 31, the results of several recent
researches by radar and radio methods into iono-
spheric ionization effects produced by meteors were
described. The $E$-layer of the ionosphere, which
occurs in a region around a height of 100 km., is
characterized by one regular and two irregular forms
of ionization. The regular ionization, the normal
$E$-layer, is known to be controlled in a uniform
manner by ultra-violet radiation from the sun. The
problems of the origin and control of the irregular
forms, which for many years have been largely a
matter for speculation, were the subject of the papers
presented at the meeting. In 1932 , A. M. Skellett
in the United States had suggested that meteors
were an important agency in producing irregular
ionospheric ionization, and with J. P. Schafer and
W. M. Goodall he gave experimental evidence of
certain increases in ionization which could be
associated with meteors. Although the experimental
data which accrued in subsequent years appeared on
the whole to support Skellett's suggestions, it is only
by the recent work, particularly in Great Britain,
that a more comprehensive account of the complex
characteristics of the irregular ionization in relation
to the meteoric theory could be given.

The irregular ionization can be classified into two types, which are distinguished most clearly by the radio-frequency at which echoes may be obtained and by their duration. The abnormal or sporadic $E$-layer occurs spasmodically for minutes or hours with a critical frequency greater than, but of the same order as, that prevailing in the normal $E$-layer. On the other hand, ionization bursts are characterized by the transient radio echoes which rarely persist for many seconds, the majority lasting only a fraction of a second; and with high-power transmitters, these echoes may be observed at frequencies greatly exceeding the critical frequency of the other $E$-layers, although the number of echoes diminishes as the frequency is increased.

Sir Edward Appleton gave the opening paper at the Physical Society's January meeting, describing recent investigations carried out with $R$. Naismith for the purpose of comparing the incidence of the bursts of ionization with the abnormal $E$-layer ionization and of determining their relation both to the incidence of meteors and to the normal $E$-layer. The observation of the ionization bursts was made at $27 \mathrm{Mc} . / \mathrm{s}$. with a high-power equipment having a wide aerial beam with its axis vertical. An analysis of the diurnal variation of the rate of occurrence of the radio echoes showed that the form of the nighttime curve was almost constant, but the day-time portion was found to alter with the season of the year. A characteristic minimum was found to occur at $1800 \mathrm{hr}$. local time throughout the year, and both the rate of occurrence and duration increased as the night progressed. In the winter, the number of bursts reached a maximum at about $0500 \mathrm{hr}$. In summer, however, the increase was found to continue until the maximum rate was reached just before or at local noon. The seasonal variation in hourly rate at noon showed a maximum burst-activity in summer; but the relation is not symmetrical, the rates being greater in the latter half of the year than in the first. These facts may be explained on the hypothesis that the ionization bursts are caused by meteors, while a marked degree of solar control is exerted over the responsiveness of the upper atmosphere. Thus, the hourly rate of sporadic meteors is known to be greater after midnight than before, and greater in the second half of the year than the first. On the other hand, the sun causes the normal $E$-layer ionization to reach a maximum near noon, the ionization being greatest in summer. A combination of these two factors would account for the observed diurnal and seasonal variations in mean rate of occurrence.

The controlling influence of the normal $E$-layer is exhibited in a parallel way in the case of the abnormal $E$-layer, which, for example, during the day shows diurnal and seasonal variations of height similar to those of the maximum normal $E$-layer ionization. Further, it is known that the abnormal $E$-layer has shown increases at the time of large meteor showers. The Giacobinid shower of October 10, 1946, afforded a particularly striking example. The remarkable increase in the number of ionization bursts at this time has been reported previously ${ }^{3}$.

There is thus a general parallelism between the two phenomena of the bursts and the abnormal $E$-layer; and it is suggested therefore that, at least in temperate latitudes, meteors are the agency primarily responsible for both these forms of irregular ionization, although the ionizability of the medium exerts a very marked effect. This controlling effect is revealed in the day-time influence of the normal $E$-layer. The ionization bursts are pictured as being associated with individual meteor trails. That there are many more echoes than visible meteors is understandable, since the greater proportion of meteors may be expected to be too small to produce visible trails.

Sir Edward Appleton also referred to the observations he made with Naismith and the B.B.C. of the meteoric Doppler whistles heard in radio sets tuned to the unmodulated carrier of short-wave radio stations, which were first reported by the Indian workers, Chamanlal and Venkataraman 4 . He suggested that the changing pitch of the whistles could be accounted for by the changes in relative velocity with respect to the observer without assuming any retardation of the meteor. The resulting beatfrequency is :

$$
f=\frac{2 V}{\lambda}\left(1-\frac{R_{0}{ }^{2}}{R^{2}}\right)
$$

where $V$ is the velocity of the meteor, $\lambda$ the wavelength, $R$ the range and $R_{0}$ the perpendicular distance to the track.

J. S. Hey then gave an account of the researches he had carried out since 1944 with G. S. Stewart at the Operational Research Group, Ministry of Supply, at frequencies around $60 \mathrm{Mc} . / \mathrm{s}$. Such high frequencies limit observations to the more intense ionospheric bursts, and it was found that the transient echoes could then be more specifically related to meteors, with far less evidence of any controlling influence exerted by normal $E$-layer ionization. Thus peaks in the rate of occurrence were found to correspond to well-known meteor showers ${ }^{5}$. The height distribution was determined by means of an aerial beam with its axis vertical, due allowance being made for the effect of beam-width. The mean height of maximum occurrence was found to be about $95 \mathrm{~km}$., and 90 per cent of all echoes occurred between 
$87 \mathrm{~km}$. and $108 \mathrm{~km}$. This height agrees well with the visually observed heights of meteors and their trains.

Observations made with radio beams having their axes inclined at elevations around $45^{\circ}$ revealed the important influence of the direction of observation on the apparent rate of occurrence. In an experiment carried out with the help of A.A. Command during June and July 1945, stations at Walmer (Kent), Aldeburgh (Suffolk) and Richmond (Surrey), respectively, were set so that their aerial beams made broad intersections at heights around $100 \mathrm{~km}$. Simultaneous observation of echoes from the three stations was a rarity, thus indicating marked aspect sensitivity in the echoing sources. This was further confimmed by the diurnal variations in rate of occurrence, which were markedly different for the three stations. The aspect sensitivity was shown to be readily explained when the transient echoes are interpreted as the broadside reflexions from meteor trails, which may be expected to show aspect variations in reflexion like those for long cylinders, as was first suggested by J. A. Pierce ${ }^{6}$. Thus, as the elevation and bearing of the active radiants change with the earth's daily rotation, corresponding changes occur in the optimum direction for obtaining radio reflexions. In fact, by finding the optimum directions experimentally, it has been found possible in certain instances to derive the positions of the radiants of the most active meteor streams.

Finally, during the Giacobinid meteor shower of October 10, 1946, not only were records obtained of the broadside reflexions from meteor trains (which show relatively only small range-movements due to drifts and turbulence); but also, by improved photographic techniques, the reflexions from the ionization in the immediate vicinity of the approaching meteors were recorded in certain instances. These observations and the derivations of meteor velocities were described in detail at a meeting of the Royal Astronomical Society on December $13,1946^{7,8}$. During part of a shower, a watch for echoes at a frequency of $212 \mathrm{Mc} / \mathrm{s}$. was also maintained, and the number of echoes was about one tenth of the number on a $64 \mathrm{Mc}$./s. equipment of similar power and beam width.

Dr. E. Eastwood described the investigations of ionospheric bursts of ionization which he has con. ducted in association with $\mathrm{K}$. A. Mercer at a number of R.A.F. radar stations during 1945-46. The aerial systems gave fixed beams covering low angles of elevations over almost all bearings. Systematic observations, made over nineteen months at the Bawdsey station on a frequency of about $23 \mathrm{Mc} / \mathrm{s}$., showed that the rate of occurrence of bursts follows a diurnal cycle with peaks at approximately $0400 \mathrm{hr}$. and $1200 \mathrm{hr}$. G.M.T. and a pronounced minimum in the afternoon. The precise form of the diurnal cycle, however, proved to be dependent on the season. The relative amplitude of the two peaks varied seasonally, but the afternoon minimum was found to be a feature of all the daily curves. The change in daily volume of activity showed an annual cycle with a maximum in the autumn and a minimum in the spring. These experimental results, as in the case of those obtained by Sir Edward Appleton and R. Naismith, were therefore in accord with the theory that attributes the bursts to ionization by sporadic meteors in an atmospheric medium subject to a marked degree of solar control.

The height of the bursts was determined from the minima in the histograms of range-distribution.
These minima could be related to the minima of the aerial beam pattern which occur at several elevations as a consequence of the interference between the direct wave and that reflected from the ground. It was concluded that most ionization bursts are produced within a thin layer located at a height of approximately $85 \mathrm{~km}$.

The magnitudes of the echoes were studied by using a single dipole asrial system. The derivation of the distribution function for the magnitudes showed that, for a given frequeney, the number of bursts per unit area per unit time having equivalent echoing areas between $A$ and $A+\delta A$ is proportional

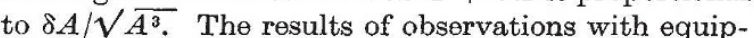
ments operating in the frequency bands $20 / 30$ and $40 / 50 \mathrm{Mc} . / \mathrm{s}$. indicated that the number of bursts was also inversely proportional to the square of the frequency.

An examination of the burst-activity for local variations was made by comparing the results at different stations in England and Scotland. Although at any given time random fluctuations were found from place to place, the average activity was constant over wide areas. Further, the results obtained during the partial solar eclipse of July 1945 did not reveal any influence on the phenomenon of burstactivity.

Dr. A. C. B. Lovell described the investigations at a frequency of $73 \mathrm{Mc} . \mathrm{s}$. which he had carried out with C. J. Banwell and J. A. Clegg in continuation of the observations of the Perseids and Giacobinids described before the Royal Astronomical Society on December 13, $1946^{8}$. By using the narrow-beam aorial system maintained at right angles to the direction of the radiant, the main characteristics of the 1946 Leonids, Orionids, Taurids, Geminids and 1947 Quadrantids were measured. The most important results were as follows :

(1) In the two richest showers (Geminids and Quadrantids), a striking correlation of hourly rate with radiant altitude was observed. The rate remained low (5-10 per hour) until the radiation attained an altitude of about $40^{\circ}$, when it increased suddenly to about 45 per hour for the Geminids and to about 60 per hour for the Quadrantids. The reverse effect was observed when the radiant was setting.

(2) Although the velocities of these meteor showers are known from visual observations to vary over a wide range, the distribution of durations of the echoes from all these five showers was identical. 60 per cent of all echoes in the showers lasted for less than 0.25 sec. Only 1 per cent had durations in excess of $10 \mathrm{sec}$. This result is important in the development of the theory of the decay of the meteor ionization, since it implies that the height at which the ionization is formed is not the principal factor determining the duration of the echo.

(3) The distribution in intensity of ionization for the Geminids and Quadrantids was calculated using formulæ given previously ${ }^{8}$. In each case the distribution was the same as that for the 1946 Giacobinids, namely, that the number of occurrences of ionization of a given intensity was inversely proportional to the intensity. In conjunction with Herlofson's theory of meteor ionization, this shows that the number of meteors of a given size is inversely proportional to the size of the meteors. This result agrees with the visual estimates that there is the same total mass of meteoric material in each magnitude. 
The work of Chamanlal and Venkataraman ${ }^{4}$ was further described by G. R. M. Garratt who, during a recent visit to New Delhi, was given an opportunity of listening to the meteoric whistles, which were first reported by the Indian workers when listening to the short-wave transmitters of All India Radio. The whistles normally lasted about $2-3$ sec., fell rapidly in pitch to zero or sometimes died away before reaching zero, and only in rare cases did the whistle reappear as an ascending note after passing through zero. Chamanlal and Venkataraman considered that these characteristics could be explained on the assumption that they were due to interference between the directly received ground-wave from the transmitter and the reflected waves which have undergone a Doppler change of frequency owing to the movement of the reflecting surface. The velocities deduced were similar to those of meteors, and the suggestion that the reflexions were associated with meteors was confirmed by direct correlation between the occurrence of a whistle and the arrival of a visible meteor. Mr. Garratt outlined the conditions for satisfactory reception of the meteoric whistles; namely, a high-power transmitter radiating an unmodulated signal at a frequency of the order of 5-15 Mc./s., and a highly sensitive receiver located within the skip distance in a position such that a very weak ground-wave is received from the transmitter. The whistle effects can be heard on almost any night of the year, the greatest number being observed about 4 a.m.

Finally, the recent investigations in the United States by J. A. Pierce ${ }^{9}$ were outlined by K. R. Eldridge. During the Giacobinid shower of October 10,1946 , about 3,500 Doppler whistles were recorded. Pierce considered that the Doppler effect is produced by an 'ionic bow-wave', the pitch being a function of both the velocity of the meteor and the rate of diffusion of the electrons. The pitch is presumed to fall from the first, because the growth of the cloud of ions is most rapid at its initial height, and goes to zero when recombination prevents further expansion of the boundary.

Correlations between the whistles and visually observed meteors were obtained when weather conditions permitted. In the case of two whistles, which were so loud that the visual watchers out-ofdoors $40 \mathrm{ft}$. away could hear them, it was noted that the whistle was heard within the first quarter of the meteor's course, and the tone fell in pitch throughout the period of the meteor's visibility.

Pierce concluded from the average number of whistles observed that his limit of sensitivity corresponded to a third-magnitude meteor. $\mathrm{He}$ calculated that the average rate of flow of energy in the four hours during which the Giacobinid shower was observed corresponded to a figure of 3 watts per sq. $\mathrm{km}$. By assuming that the energy was expended in a layer $30 \mathrm{~km}$. thick, and that 15 electron volts are required per ion, it was calculated that the ion density would be of the same order as the maximum density of ionization in the normal $E$-layer at the sub-solar point.

J. S. Hex

${ }^{1}$ Skellett, Proc. Inst. Rad. Eng., 20, 1933 (1932).

${ }^{2}$ Schafer and Goodall, Proc. Inst. Rad. Eng., 20, 1941 (1932).

${ }^{3}$ Appleton and Naismith, Nature, 158, 936 (1946).

4 Chamanlal and Venkataraman, Electrotechnics, 14, 28 (1941).

${ }^{5}$ Hey and Stewart, Nature, 158, 481 (1946).

' Pierce, Proc. Inst. Rad. Eng., 26, 892 (1938).

${ }^{7} \mathrm{Hey}$, Parsons and Stewart, Mon. Not. Roy. Astro. Soc., in the press.

8. See Nature, 159, 119 (1947).

${ }^{9}$ Pierce, Phys. Rev., 71, 88 (1947).

\section{METEORS, COMETS AND METEORIC IONIZATION}

$\mathrm{O}^{\mathrm{N}}$ March 19 and 20 a conference on meteors, comets and meteoric ionization was held in the University of Manchester Physical Laboratories under the auspices of the Physical Society. The chairman, Prof. P. M. S. Blackett, in opening the conference, remarked that this was probably the first conference of its kind to be held. A widespread interest in meteors and comets is arising because of the new radio methods of detecting the ionization due to meteors in the atmosphere. Results of importance to astronomers and physicists are now being obtained; and it was hoped that this joint gathering would be of benefit to both.

\section{Meteors}

In the first lecture, J. P. M. Prentice, director of the Meteor Section of the British Astronomical Association, described the general background of our knowledge of meteors. The following is a brief abstract of his remarks.

(A) Numbers. The major streams exert a dominant influence on the curve of annual variation, and the rate of occurrence of a given stream is a function of the radiant altitude. The general level of meteor activity after eliminating the major streams is rather low, about 4-10 an hour, and shows daily and annual variations due mainly to the concentration of radiants around the apex of the earth's path. A particular observer sees only a small fraction of the fainter meteors appearing in his zone of vision, so that although the observed numbers fall off after magnitude $4 \cdot 5$, the real numbers continue to increase by a factor $2 \cdot 3$ per magnitude. Beyond the limits of naked-eye visibility, the number of telescopic meteors increases by the same factor at least down to magnitude 10 and probably far beyond.

(B) Radiants. The divergence of meteors from radiant points is an effect of perspective, showing that the motions of those meteors are parallel and that they form members of one stream. These radiants are apparent radiants, due to the combined orbital velocities of the meteors and the earth. Some twelve major streams are known, of which eight appear annually; in addition, there are many minor streams of varying degrees of tenuousness, and some meteors pursuing individual paths in space properly called 'sporadic'. A very few streams are known from the similarity of their orbital elements to be associated with comets, although the proof is only complete for the three instances (possibly four) in which the periods have been determined. The majority of radiants are not points but large areas of radiation some $10^{\circ}-15^{\circ}$ in diameter.

(C) Paths in the Atmosphere. The meteor zone lies generally at an altitude between $125 \mathrm{~km}$. and $70 \mathrm{~km}$., though exact limits cannot be assigned. The height of appearance and disappearance is determined primarily by the entrance velocity, although the beginning height is also correlated with the zenithal magnitude. This difference in height is generally a function of zenithal magnitude only, being sensibly independent of velocity and of radiant altitude except for very low radiant altitudes. The heights are determined by simultaneous observations, which also yield the path-length $P$ and the duration of flight $t$, and hence the obsirved velocity $P / t$. There is some observational evidence of a general decelera- 